\title{
Quistes subglóticos adquiridos: Reporte de tres casos y revisión de la literatura
}

\section{Acquired subglottic cysts: Report of three cases and review of the literature}

\author{
Francisco Pérez T. ${ }^{1,2}$, Lorena Aguayo G. ${ }^{2}$, Daniela Gutiérrez C. ${ }^{2}$, Maya Kuroiwa ${ }^{2,3}$, \\ Cecilia Sedano $M^{2}{ }^{2}$, Natalia Bahamondes M. ${ }^{1,2}$, Andrés Alvo $V^{1,2,4}$
}

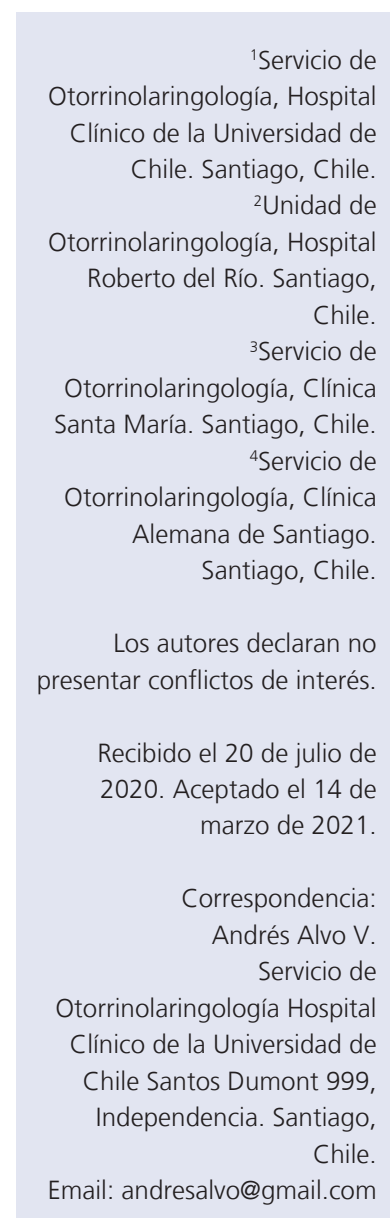

\section{Introducción}

Los quistes subglóticos fueron descritos por primera vez por Wigger y Tang el año 1968, siendo presentados como una causa rara de

\section{Resumen}

Los quistes subglóticos adquiridos son una causa rara de estridor en la infancia, cuyo reporte ha ido en aumento en las últimas décadas. Su aparición se relaciona con la prematurez y la intubación en el periodo neonatal. Histológicamente, se observa una obstrucción de las glándulas mucosas de la subglotis debido una metaplasia escamosa del epitelio respiratorio. Esta es una condición que usualmente requiere de un alto índice de sospecha para llegar al diagnóstico, ya que pueden confundirse con otras patologías como laringitis aguda (croup), laringomalacia o asma. La nasofibroscopía permite sospechar su presencia, pero el gold standard diagnóstico lo constituye la laringo-traqueo-broncoscopía directa en pabellón. Existen diversas técnicas para su manejo, siendo las más frecuentemente utilizadas la marsupialización con instrumental frío y láser. La recurrencia es frecuente, por lo que algunos autores han utilizado mitomicina $\mathrm{C}$ y la terapia antirreflujo para intentar disminuirla. Sin embargo, hasta la fecha falta evidencia de calidad que permita llegar a un consenso respecto al manejo ideal de esta patología. En este trabajo, presentamos tres casos clínico de pacientes con antecedentes de prematurez que fueron diagnosticados con quistes subglóticos adquiridos y manejados en un hospital pediátrico de alta complejidad.

Palabras clave: Quistes subglóticos adquiridos, prematurez, intubación.

\begin{abstract}
Acquired subglottic cysts are an infrequent cause of stridor in infants, which has been increasingly reported in the last decades. Its appearance is related to prematurity and intubation in the neonatal period. Histologically, findings are characterized by an obstruction of the mucosal glands, due to squamous metaplasia of the respiratory epithelium. This condition usually requires a high index of suspicion to be diagnosed, as it can be misdiagnosed as croup, laryngomalacia or asthma. Flexible nasendoscopy allows an initial exploration of the larynx, but direct laryngo-tracheo-bronchoscopy in the operating room is the diagnostic gold standard. There are several techniques for its management, but the most frequently used are cold-steel marsupialization and laser. Recurrence is common, and some authors have used mitomycin $C$ and antireflux therapy to try to decrease it. However, up to date, there is a lack of high-quality evidence, regarding the ideal management of this pathology, which prevents reaching a consensus. In this article, we present three clinical cases of premature patients who were diagnosed with subglottic cysts, treated in a tertiary pediatric hospital.
\end{abstract}

Keywords: Acquired subglottic cysts, prematurity, intubation. 
de riesgo para su aparición son la prematurez y la intubación en el periodo neonatal. Se estima una incidencia de 1,82 por cada 100.000 recién nacidos vivos para el caso de los quistes congénitos, sin existir información confiable respecto a los quistes adquiridos ${ }^{3}$, aunque se considera que estos últimos son los más frecuentes.

En las últimas décadas, la significativa mejoría en la sobrevida de recién nacidos prematuros ha llevado a un aumento en el reporte de casos, especialmente en aquellos pacientes que han requerido intubación orotraqueal con estadía en unidades de cuidados intensivos ${ }^{4}$. Sin embargo, este aumento no se ha correlacionado necesariamente con la aparición de evidencia de mayor calidad, existiendo aún falta de consenso en cuanto a su incidencia, fisiopatología y manejo ${ }^{3}$. En este trabajo presentamos tres casos de pacientes con quistes subglóticos evaluados en el Hospital de Niños Roberto del Río (HRR), destacando la presencia de los factores de riesgo ya descritos (prematurez e intubación en el periodo neonatal) en todos ellos.

\section{Caso Clínico 1}

Paciente de sexo femenino, pretérmino de 28 semanas, con antecedente de displasia broncopulmonar que requirió intubación orotraqueal por 14 días en periodo neonatal. A los tres meses de edad (un mes de edad corregida), consultó en el servicio de otorrinolaringología por cuadro de una semana de evolución de estridor bifásico que cedía con el uso de corticoides inhalatorios y salbutamol. Se realizó una nasofibroscopía que mostró una probable estenosis subglótica con compromiso parcial del lumen (Figura 1).

La paciente fue llevada a pabellón para una laringoscopía directa, observándose una disminución del calibre de la subglotis a expensas de múltiples quistes. Se realizó una dilatación con tubos orotraqueales (TOT) hasta $4 \mathrm{~mm}$ en primera instancia, con ruptura de los quistes, logrando un calibre adecuado de vía aérea. Sin embargo, la paciente presentó reaparición del estridor al día siguiente, por lo que se sometió a un segundo procedimiento donde se evidenció reaparición de los quistes de la región anterior, los cuales fueron marsupializados usando técnica fría (Figura 2a y 2b). Al finalizar el procedimiento se aplicó crema de betametasona con gentamicina (Mixgen ${ }^{\circledR}$, Laboratorio Chile). El informe anátomopatológico informó quistes ductales (Figura 3).

A las 3 semanas se realizó una nueva laringoscopía directa, donde no se observó recidiva de los quistes. La paciente se mantuvo en seguimiento por 18 meses tras el procedimiento, sin reaparición de sintomatología.

\section{Caso Clínico 2}

Paciente de sexo masculino, pretérmino de 27 semanas con antecedente de enfermedad de membrana hialina que requirió intubación orotraqueal por 12 días en el periodo neonatal. En los meses siguientes presentó dos cuadros diagnosticados como laringitis aguda (croup) que fueron manejados en forma ambulatoria. A los 8 meses de edad ( 5 meses de edad corregida), consultó por un cuadro interpretado nuevamente como croup, donde se pesquisó un estridor bifásico con dificultad respiratoria que respondió escasamente a terapia, por lo que se hospitalizó para estudio. Se realizó una nasofibroscopía que mostró una estrechez de la subglotis de aspecto quístico, que ocupaba aproximadamente el 90\% del lumen (Figura 4).

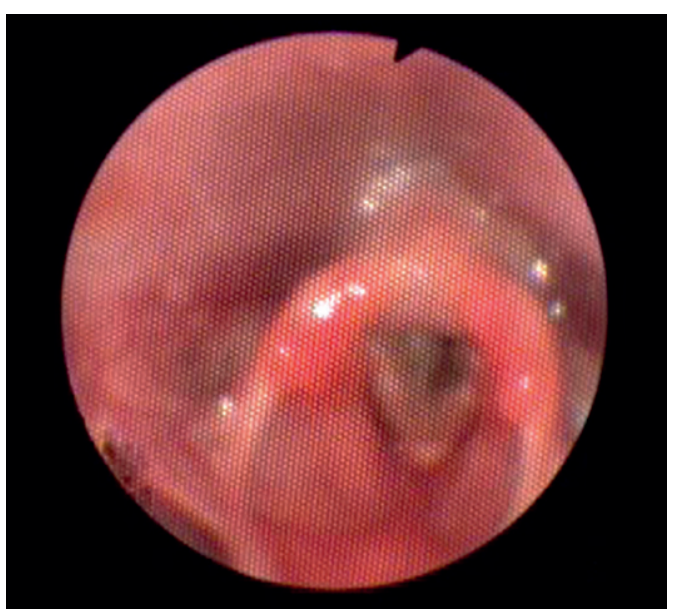

Figura 1. Caso 1, nasofibroscopía preoperatoria. 


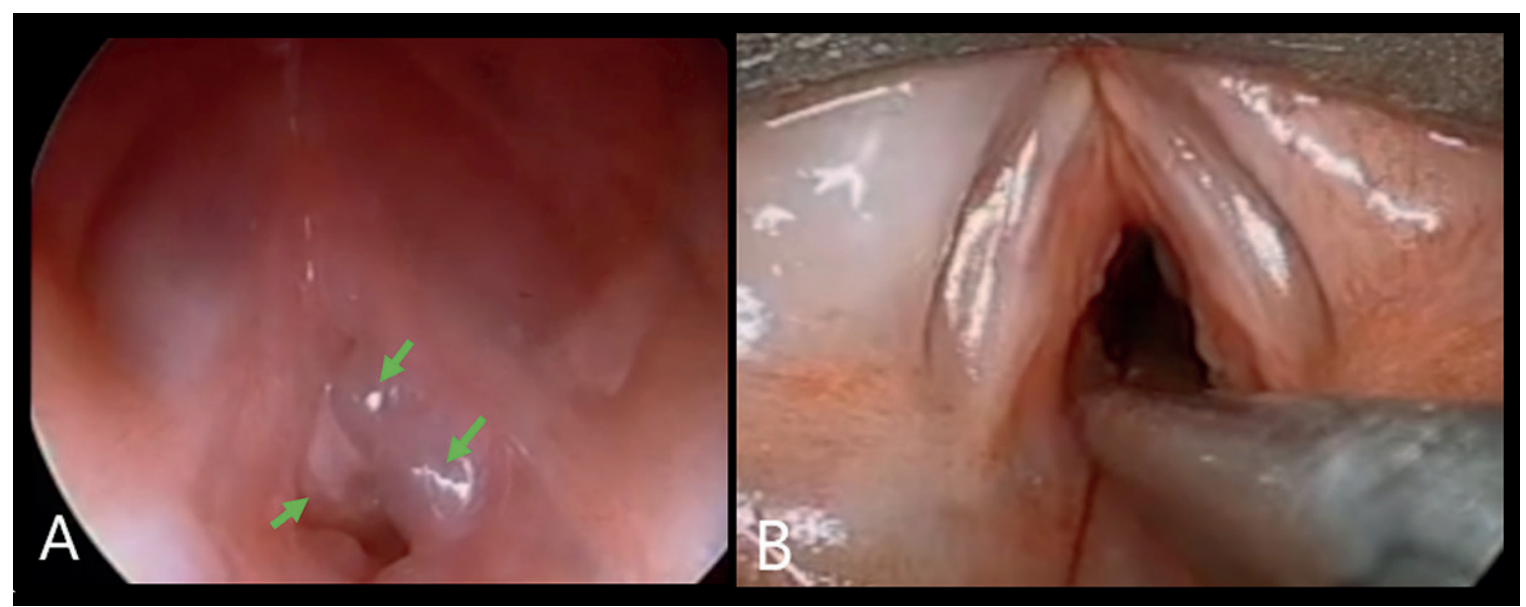

Figura 2. Caso 1, A: Laringoscopía directa donde se observan quistes subglóticos bilaterales (flechas verdes); B: Marsupialización fría de quistes con pinza en copa.

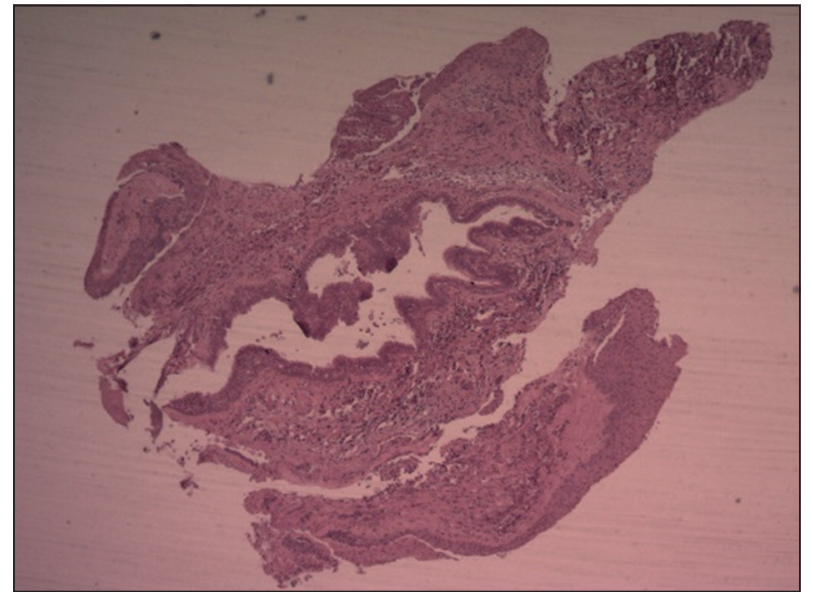

Figura 3. Caso 1, biopsia de quiste con tinción H-E. Se informa como ectasia ductal quística de glándulas mucosas laríngeas (quiste ductal).

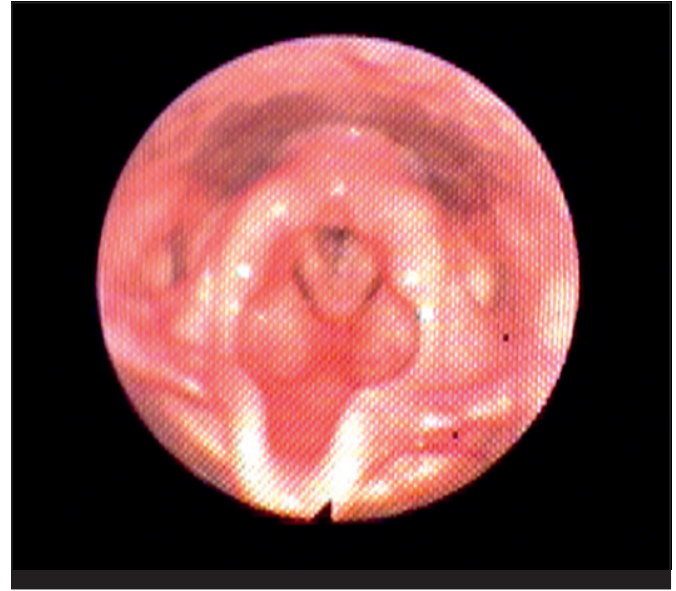

Figura 4. Caso 2, nasofibroscopía preoperatoria.

\section{Caso Clínico 3}

Paciente nacido y atendido inicialmente en otro centro de salud, de sexo masculino, pretérmino de 29 semanas, gemelar II, con antecedente de enfermedad de membrana hialina que requirió intubación orotraqueal por cuatro días. Además, se realizó una cirugía por anquiloglosia al segundo mes de vida, donde se describió una intubación dificultosa, evolucionando con estridor posextubación, el cual persistió hasta el alta de la unidad de neonatología. 
A los cinco meses de vida (tres meses de edad corregida), consultó en el servicio de urgencias de nuestro hospital por cuadro de una semana de tos seca, afebril, con estridor en aumento y dificultad respiratoria progresiva, con retracción costal y subcostal. El cuadro se interpretó como una laringitis aguda y se realizó manejo médico con mala respuesta, por lo que fue hospitalizado. En este contexto, fue evaluado por otorrinolaringología. Se realizó una nasofibroscopía que mostró una probable estenosis subglótica con compromiso parcial del lumen (Figura 5).

El paciente fue llevado a quirófano para laringoscopía directa, observándose una disminución del lumen subglótico de $80 \%$, predominantemente hacia anterior (Figura 6), el cual fue inicialmente tratado con dilataciones endoscópicas con tubos orotraqueales y broncoscopio, siendo dado de alta con discreto estridor bifásico en reposo.

El paciente evolucionó inicialmente en forma favorable, pero tras un mes presentó aumento del estridor y se realizó nueva nasofibroscopía que no fue concluyente, por lo que se decidió realizar una nueva revisión en pabellón, donde se observaron quistes subglóticos bilaterales que obstruían cerca de un 90\% del lumen, el mayor a derecha y anterior. Se realizó marsupialización fría y dilatación en tres oportunidades, durante la misma hospitalización, por recidiva de las lesiones. En el último procedimiento, se logró una vía aérea con estenosis residual de 40\%-50\%, saliendo extubado de pabellón y siendo dado de alta al día siguiente. El paciente se mantiene en controles periódicos y en el seguimiento a 6 meses no hay reaparición del estridor.

\section{Discusión}

Los quistes subglóticos son una causa infrecuente de estridor en lactantes. Se han clasificado en congénitos y adquiridos; sin embargo, dado que la prematurez es un factor de riesgo para ambos y considerando que la mayoría de los prematuros extremos presenta dificultad respiratoria que requiere intubación, se vuelve difícil diferenciarlos ${ }^{2,5}$. Si bien no existen datos confiables para estimar la incidencia de quistes subglóticos ${ }^{3}$, se piensa que la gran mayoría de

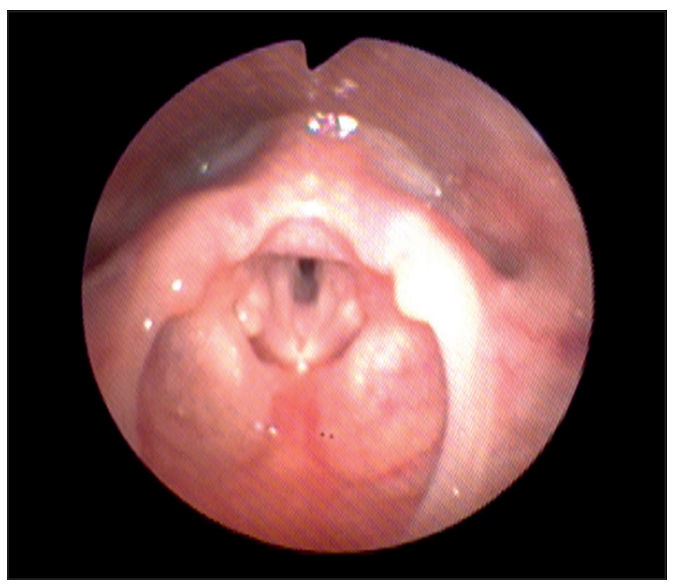

Figura 5. Caso 3, nasofibroscopía preoperatoria. Se observa una disminución del lumen subglótico, sin verse claramente imágenes quísticas.

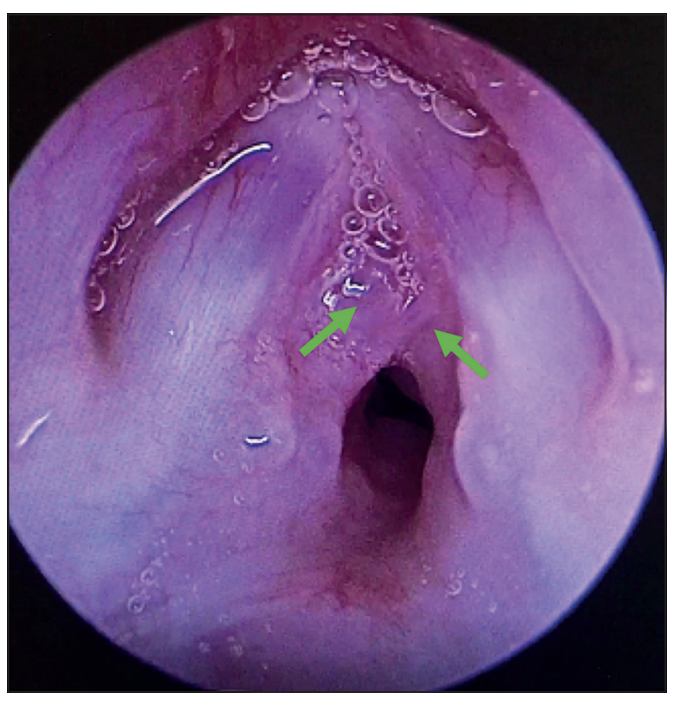

Figura 6. Caso 3, laringoscopía directa donde se observan quistes subglóticos anteriores bilaterales (flechas verdes).

estos quistes son adquiridos, siendo la historia clásica un estridor bifásico progresivo que aparece meses después de una intubación en un recién nacido prematuro.

En un reporte, Rodríguez y cols. mencionan que en el 7\% de los lactantes con estridor sometidos a laringoscopía directa o broncoscopía se observaron quistes subglóticos ${ }^{4}$, mientras que en otro estudio se menciona como la cuarta causa más frecuente de obstrucción de 
vía aérea ${ }^{6}$. Sin embargo, esta alta frecuencia relativa no es lo comúnmente descrito en la literatura, reportándose más bien como casos aislados o pequeñas series de casos.

Los principales factores de riesgo para el desarrollo de quistes subglóticos descritos en la literatura son la prematurez y la intubación temprana ${ }^{2,7}$. Además, la patología intercurrente (sepsis, desnutrición), el reflujo gastroesofágico y la cicatrización patológica, son factores que favorecen su formación ${ }^{1,7}$. Otros autores describen mayor frecuencia de quistes en género masculino que femenino, aunque puede ser explicado porque los varones tienen además mayor riesgo de prematurez $z^{5,6}$. Otros factores de riesgo mencionados en la literatura son la intubación repetida, traumática o con tubo de calibre inadecuado. En un estudio, se comparó la intubación nasotraqueal y orotraqueal, sin evidencia de que el modo de intubación tenga relación alguna con la incidencia o el sitio de aparición de los quistes ${ }^{2,5-7}$. A su vez, hay estudios que mencionan aparición de quistes con intubaciones desde menos de 24 horas, por lo que, hasta el momento, parece no haber una clara relación entre la duración de la intubación y la aparición de quistes ${ }^{6,8}$.

Considerando que la intubación, por lo general, es realizada por un profesional diestro, y dada la conformación del laringoscopio, la región posterior e izquierda de la subglotis son las zonas más susceptibles a traumatismos durante la intubación, acorde con los sitios más frecuentes de aparición de estos quistes reportados en la literatura ${ }^{6}$. Se mencionan como posibles eventos traumáticos el efecto de "pistón neumático" que se produce entre el tubo y el ventilador, así como la intubación repetida, la succión endotraqueal o el desplazamiento del tubo. Además, el hecho de que los niños tengan mayor número de glándulas submucosas en la subglotis puede aumentar su predisposición a la formación de quistes ${ }^{2,5}$.

A nivel histopatológico, inicialmente se produce un desgarro o ulceración en la mucosa, seguido de necrosis, reparación tisular con formación de tejido de granulación y posterior regeneración del epitelio. Sin embargo, en aquellos pacientes que desarrollan quistes, se postula que el tejido de granulación conduce a la formación de fibrosis subepitelial y metaplasia escamosa, los que producen una posterior obstrucción de las glándulas mucosas y llevan finalmente a la formación de quistes ${ }^{2,4,7,9}$.

La edad más frecuente de presentación es entre los 4-6 meses de vida ${ }^{3}$, lo cual concuerda con la edad de presentación observada en nuestros pacientes. Por lo general, se describe la aparición de quistes dentro de los 12 meses siguientes a la intubación ${ }^{2,5}$. En un estudio, se describe un promedio de 8,2 meses entre la intubación y el diagnóstico, con un promedio de dos semanas entre la aparición de síntomas y el diagnóstico nasofibroscópico, con sólo un caso que tardó 12 meses al ser diagnosticado erróneamente como asma ${ }^{1}$.

La mayor parte de los autores menciona el estridor como el síntoma más frecuente de presentación, siendo descrito en seis de nueve casos por Aksoy y cols. y en 11 de 14 pacientes por Watson y cols. ${ }^{2,5,6}$. Esto concuerda con la serie presentada, en que el estridor fue el síntoma de presentación en todos los pacientes. Sin embargo, una serie de Halimi y cols., describe como síntoma más frecuente de presentación la disnea con retracción supraesternal (82,3\%), seguida por estridor bifásico $(71 \%)$ y distrés respiratorio con desaturación $(30 \%)^{1}$. Otros autores describen también episodios apneicos, dificultad en la alimentación, cambios en la voz posextubación y tos "disfónica" como forma de presentación ${ }^{2,4}$.

Muchas veces, los quistes subglóticos son confundidos con otras patologías como laringomalacia, asma o laringitis ${ }^{4}$, por lo que se vuelve sumamente importante conocerlos y sospechar su presencia para evitar importantes errores diagnósticos. Por esto, se plantea que debe sospecharse la presencia de quistes subglóticos en pacientes con antecedentes de prematurez y/o intubación, y que se presenten con estridor recurrente, en quiénes se recomienda realizar prontamente estudio dirigido para acelerar el diagnóstico y tratamiento ${ }^{1,4}$.

El diagnóstico se realiza mediante videolaringoscopía o laringo-traqueo-broncoscopía rígida. Las imágenes no se solicitan de manera rutinaria. La tomografía computarizada (TC) sólo permite visualizar el lumen de la vía aérea, sin aportar datos sobre la estructura de su pared, aunque la resonancia magnética (RM) podría entregar algo más de información al respecto ${ }^{3}$. El tratamiento quirúrgico debe ser reservado para lesiones que disminuyan el 
lumen subglótico en forma significativa. En caso de haber quistes bilaterales, a veces se recomienda un tratamiento secuencial para prevenir una estenosis cicatricial ${ }^{1}$.

En cuanto a las técnicas quirúrgicas, se ha descrito la marsupialización con instrumental frío, láseres de diodo y $\mathrm{CO}_{2}$ y el uso de microdebridador como alternativas. Algunos autores consideran la técnica fría como de elección, pues evita el daño térmico de la mucosa sana y podría presentar menor tasa de recurrencia, aunque falta evidencia para poder respaldar estas observaciones. En cuanto al láser, algunos autores recomiendan el diodo por sobre $\mathrm{CO}_{2}$, ya que genera menor daño térmico de la mucosa adyacente, es más fácil de utilizar en vías aéreas pequeñas y ha demostrado ser una técnica efectiva para el tratamiento de quistes subglóticos en población pediátrica ${ }^{3,10}$. Por otra parte, el microdebridador permite un mejor control durante la escisión del quiste dado que realiza succión concomitante; sin embargo, el tamaño del instrumental dificulta su uso en la infancia temprana, además del riesgo de sangrado inherente a la técnica ${ }^{1}$. En un estudio, además del manejo del quiste con técnica fría o láser $\mathrm{CO}_{2}$, se realizó dilatación con balón a todos los pacientes, sin presentar recurrencias ${ }^{5}$.

En cuanto a nuestros casos, la exposición quirúrgica se logra mediante laringoscopía de suspensión o bien con la ayuda de un videolaringoscopio (C-MAC ${ }^{\circledR}$, Karl Storz, Tuttlingen, Alemania). Aunque el uso del microscopio quirúrgico permite visión en profundidad y el uso de ambas manos para operar, en general optamos por el uso de endoscopios de $0^{\circ}$ y 4 $\mathrm{mm}$ por la mejor calidad de imagen y visión panorámica. Los quistes fueron marsupializados usando pinzas de copa o con microtijeras. Los procedimientos son realizados bajo anestesia general, idealmente con el paciente en ventilación espontánea o, de no ser posible, en apnea intermitente, compartiendo la vía aérea con el anestesista.

La recurrencia de quistes subglóticos es alta, siendo descrita entre un 19\%-70\% de los casos según distintos autores, en especial en los primeros cuatro meses posteriores a la resolución quirúrgica, pero pudiendo ocurrir por un periodo de hasta dos años ${ }^{4-6}$. En un estudio, se menciona que puede existir relación entre la presencia de múltiples quistes al momento del diagnóstico con una mayor tasa de recurrencia ${ }^{2}$. Además, está descrito que la recurrencia no siempre ocurre en el mismo sitio inicial, lo que puede indicar que existe un proceso patológico continuo subyacente.

Se recomienda reevaluar la vía aérea en un plazo de 2-4 meses, dado que la mayor parte de la recurrencia parece ocurrir en este periodo de tiempo, manteniendo posteriormente al paciente en control clínico ${ }^{6}$. Algunos autores han utilizado mitomicina-C en forma tópica como terapia adyuvante para prevenir la recurrencia y evitar la cicatrización excesiva, con resultados promisorios, aunque la evidencia es escasa como para recomendar su uso rutinario. También, es importante tener en consideración los potenciales riesgos asociados (daño renal, fibrosis pulmonar), aunque no se hayan reportado mediante uso tópico del agente $e^{2,4}$.

Se ha observado también una relación entre quistes subglóticos y reflujo gastroesofágico; se plantea que el reflujo pudiera ser secundario al aumento de la presión negativa intratorácica debido a la obstrucción de vía aérea, similar a lo que ocurre en pacientes con laringomalacia. Sin embargo, pese a que no hay una relación de causalidad demostrada, se recomienda mantener tratamiento antirreflujo durante el periodo previo y posterior a la cirugía por tiempo prolongado, como un factor que pudiera ayudar a disminuir la alta tasa de recurrencia ${ }^{1,5}$.

\section{Conclusión}

Los quistes subglóticos adquiridos son una causa infrecuente de estridor en lactantes. Se desconoce su real incidencia, aunque pareciera ir en aumento dada la mayor sobrevida de recién nacidos prematuros. Los principales factores de riesgo descritos son la intubación temprana y la prematurez. Es importante tener una alta sospecha clínica para lograr un diagnóstico adecuado e implementar un manejo oportuno, evitando morbilidad asociada. Si bien no existe consenso, parece ser que la técnica fría o el láser de diodo, con o sin dilatación endoscópica, son el manejo de elección en la actualidad. Existe una alta tasa de recurrencia descrita en la literatura, por lo que se debe realizar seguimiento por un periodo de tiempo prolongado, aunque no existe consenso respec- 
to a la duración de éste. El uso de mitomicina C tópica y la terapia antirreflujo podrían cumplir un rol en disminuir la recidiva, aunque falta evidencia para recomendar su uso rutinario.

\section{Bibliografía}

1. Halimi C, Nevoux J, Denoyelle F, Garabedian EN, Leboulanger N. Acquired subglottic cysts: Management and long term outcome. Int J Pediatr Otorhinolaryngol. 2012;76(4):589-592. doi: 10.1016/j. ijporl.2012.01.023.

2. Agada FO, Bell J, Knight L. Subglottic cysts in children: A 10-year review. Int J Pediatr Otorhinolaryngol. 2006;70(8):1485-1488. doi: 10.1016/j.ijporl.2006.03.010.

3. Villarino R. Quiste subglótico adquirido como causa de estridor recurrente en un lactante. Acta Pediatr Esp. 2019;77(5-6):104-106.

4. Rodrigues AJ, Cardoso SR, Ricci Cereda DC, Peçanha Gonçalves ME. Subglottic cyst: a rare cause of laryngeal stridor. J Bras Pneumol. 2012;38(1):138-139. doi: 10.1080/13518040701205365.
5. Aksoy EA, Elsürer C, Serin GM, Ünal ÖF. Evaluation of pediatric subglottic cysts. Int J Pediatr Otorhinolaryngol. 2012;76(2):240-243. doi: 10.1016/j. ijporl.2011.11.012.

6. Watson GJ, Malik TH, Khan NA, Sheehan PZ, Rothera MP. Acquired paediatric subglottic cysts: A series from Manchester. Int J Pediatr Otorhinolaryngol. 2007;71(4):533-538. doi: 10.1016/j. ijporl.2006.11.014.

7. Álvarez Neri H, Blanco Rodríguez G, Vega Rodríguez A, Teyssier Morales G, Ortiz Moreno C, Morera Serna E. Tratamiento quirúrgico de los quistes subglóticos adquiridos. Acta Otorrinolaringol Esp. 2013;64(5):371-373. doi: 10.1016/j. otorri.2012.02.006.

8. Smith SP, Berkowitz RG, Phelan PD. Acquired Subglottic Cysts in Infancy. Arch Otolaryngol Head Neck Surg. 1994;120:921-924.

9. Smith JD, Cotton R, Meyer CM. Subglottic Cysts in the Premature Infant. Arch Otolaryngol Neck Surg. 1990;116(4):479-482. doi: 10.1001/ archotol.1990.01870040101023.

10. Miró I, Gutiérrez C, Fonseca R, et al. Manejo multidisciplinar de la patología subglótica: revisión a 5 años. Cir Pediatr. 2018;31(2):66-70. 